\title{
Six-dimensional correction of intra-fractional prostate motion with CyberKnife stereotactic body radiation therapy
}

\author{
Siyuan Lei ${ }^{1}$, Nathaniel Piel ${ }^{1}$, Eric K. Oermann ${ }^{1}$, Viola Chen ${ }^{1}$, Andrew W. Ju ${ }^{1}$, Kedar N. Dahal ${ }^{1}$, \\ Heather N. Hanscom ${ }^{1}$, Joy S. Kim ${ }^{1}$, Xia Yu ${ }^{1}$, Guowei Zhang ${ }^{1}$, Brian T. Collins ${ }^{1}$, Reena Jha ${ }^{2}$, \\ Anatoly Dritschilo ${ }^{1}$, Simeng Suy ${ }^{1}$ and Sean P. Collins ${ }^{1}{ }^{*}$
}

${ }^{1}$ Department of Radiation Medicine, Georgetown University Hospital, Washington, DC, USA
${ }^{2}$ Department of Radiology, Georgetown University Hospital, Washington, DC, USA

\section{Edited by:}

Joe O'Sullivan, Queen's University

Belfast, UK

Reviewed by:

Alan Hounsell, Belfast Health and

Social Care Trust, UK

Nicholas Van As, Royal Marsden

Hospital, UK

*Correspondence:

Sean P. Collins, Department of Radiation Medicine, Georgetown

University Medical Center, 3800

Reservoir Road, Northwest,

Washington, DC 20007, USA.

e-mail: spc9@georgetown.edu
Large fraction radiation therapy offers a shorter course of treatment and radiobiological advantages for prostate cancer treatment. The CyberKnife is an attractive technology for delivering large fraction doses based on the ability to deliver highly conformal radiation therapy to moving targets. In addition to intra-fractional translational motion (left-right, superior-inferior, and anterior-posterior), prostate rotation (pitch, roll, and yaw) can increase geographical miss risk. We describe our experience with six-dimensional (6D) intra-fraction prostate motion correction using CyberKnife stereotactic body radiation therapy (SBRT). Eighty-eight patients were treated by SBRT alone or with supplemental external radiation therapy. Trans-perineal placement of four gold fiducials within the prostate accommodated X-ray guided prostate localization and beam adjustment. Fiducial separation and non-overlapping positioning permitted the orthogonal imaging required for $6 \mathrm{D}$ tracking. Fiducial placement accuracy was assessed using the CyberKnife fiducial extraction algorithm. Acute toxicities were assessed using Common Toxicity Criteria v3. There were no Grade 3, or higher, complications and acute morbidity was minimal. Ninety-eight percent of patients completed treatment employing $6 \mathrm{D}$ prostate motion tracking with intra-fractional beam correction. Suboptimal fiducial placement limited treatment to 3D tracking in two patients. Our experience may guide others in performing 6D correction of prostate motion with CyberKnife SBRT.

Keywords: intra-factional, prostate motion, CyberKnife, six-dimensional, hypo-fractionated radiation therapy and fiducial placement

\section{INTRODUCTION}

Current radiation therapy options for treating clinically localized prostate cancers include intensity-modulated radiation therapy (IMRT), low-dose-rate (LDR) brachytherapy, high-dose-rate (HDR) brachytherapy, proton beam therapy, and stereotactic body radiation therapy (SBRT; Collins et al., 2011). Recent trends for improving local control and patient quality of life, have focused on dose escalation (Pollack et al., 2002; Zelefsky et al., 2002) and on hypo-fractionation (Fowler, 2005; Miles and Lee, 2008). Such treatment requires smaller treatment margins and greater precision to limit geographic misses.

Technical factors of radiation delivery can contribute substantially to local failure and excessive normal tissue toxicity. Patient positioning errors and prostate motion limit the ability to accurately deliver radiation therapy to the prostate. Pelvic immobilization devices and daily set-up verification decrease, but do not eliminate, patient repositioning errors (Bentel et al., 1995). Due to rectal and bladder filling, prostate gland position has been shown to vary on a day-to-day basis (inter-fraction motion) and within a treatment session (intra-fraction motion; Kupelian et al., 2008). This motion is commonly largest in the anterior-posterior and inferior-superior directions (Crook et al., 1995; Dawson et al., 1998; Langen et al., 2008). To accommodate such uncertainties, a
$0.5-$ to $1-\mathrm{cm}$ margin is commonly added to the clinical treatment volume (CTV, the prostate, and proximal seminal vesicles) to generate a planning treatment volume (PTV; Litzenberg et al., 2006). The large size of this PTV margin, however, limits the extent of safe dose escalation and hypo-fractionation (Pollack et al., 2002; Zelefsky et al., 2002; Fowler, 2005; Miles and Lee, 2008).

Several prostate localization techniques have been utilized to minimize PTV margins, including trans-abdominal ultrasound (Chandra et al., 2003) and in-room kilovoltage CT (Moseley et al., 2007). More commonly, intra-prostatic fiducial localization with $\mathrm{X}$-ray imaging is employed to correct for inter-fraction prostate translations (inferior to superior, left to right, and anterior to posterior; Kupelian et al., 2005). Fiducial migration during the course of therapy is rare (Kupelian et al., 2005) and prostate deformation is minimal ( $<1 \mathrm{~mm}$; Deurloo et al., 2005; Van Der Wielen et al., 2008) making intra-prostatic fiducials an excellent surrogate for prostate location. The technique offers the significant advantage of ease of use and reproducibility (Ullman et al., 2006). Its implementation for daily isocenter localization and set-up reduces required PTV margins (Litzenberg et al., 2006). Correction of intra-fraction prostate translations further decreases the required margins to less than $3 \mathrm{~mm}$ (Litzenberg et al., 2006). 
In addition to prostate translational motion, prostate rotation around the three translational axes (pitch, roll, and yaw; Kaiser et al., 2006) can non-uniformly increase the required PTV margins (Langer et al., 2005; Li et al., 2009). Rotational corrections are especially important when the CTV includes the seminal vesicles which can be significantly under-dosed without such corrections (Lips et al., 2009; Rijkhorst et al., 2009). Currently, most radiation delivery systems correct only for prostate translations. While this may be acceptable when using large PTV margins, failure to correct for rotations with the small PTV margins required for hypo-fractionation may result in under-dosing of the CTV.

Hypo-fractionated SBRT using the CyberKnife (Accuray Incorporated, Sunnyvale, CA, USA) is a recent technical improvement for treating clinically localized prostate cancer (Fuller et al., 2008; Friedland et al., 2009; Katz et al., 2010b; Freeman and King, 2011; Oermann et al., 2011; Jabbari et al., in press; King et al., in press). In general, $35-40 \mathrm{~Gy}$ is delivered to the prostate in four to five fractions. CyberKnife SBRT precisely delivers highly conformal radiation to the prostate with six-dimensional (6D) correction of intra-fraction prostate motion (Xie et al., 2008; Kilby et al., 2010). Orthogonal X-ray images are used to locate gold fiducial markers within the prostate and allow for targeting and correction of the therapeutic beam during an individual treatment. Fiducials allow tracking and position correction of prostate motion in 6D (Figure 1A). Here, we describe our experience with $6 \mathrm{D}$ correction of intra-fraction prostate motion with CyberKnife SBRT.

\section{MATERIALS AND METHODS}

This is an Institutional IRB-approved retrospective review of 88 patients treated with SBRT with or without supplemental external radiation therapy from July 2008 to July 2010. This analysis was limited to patients whose fiducials were placed by the transperineal approach. Fiducials were utilized for both SBRT and conventional radiation therapy in patients receiving supplemental external radiation therapy (Katz et al., 2010a; Oermann et al., 2010).

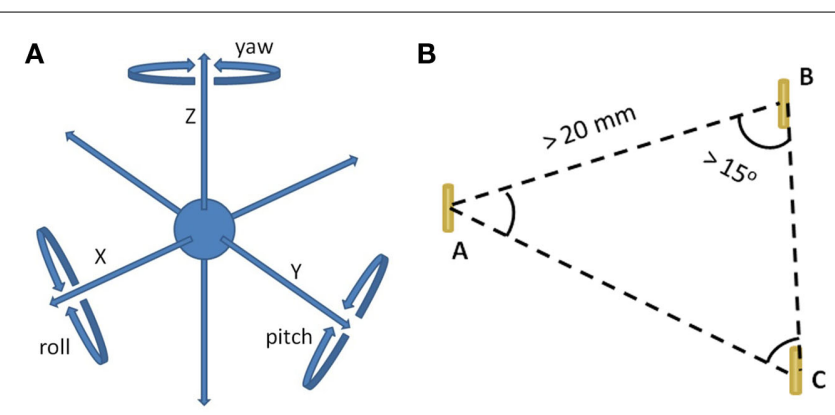

FIGURE 1 | (A) The 6D of possible prostate motion. The $X-, Y$-, and $Z$-axes represent translational motion, while pitch, roll, and yaw represent rotation around each of these axes. (B) Fiducial placement criteria for 6D tracking. (1) A minimum of three fiducials are implanted within the prostate. (2) The distance between any two fiducials must be greater than $20 \mathrm{~mm}$ (Spacing threshold). (3) All angles formed by the fiducial triplets must be greater than $15^{\circ}$ (Co-linearity threshold).

\section{TRANS-PERINEAL FIDUCIAL PLACEMENT}

Fiducial implantation for CyberKnife prostate treatment is technically similar to fiducial placement for other forms of external beam radiation therapy (Henry et al., 2004; Moman et al., 2010), albeit with several key differences. A minimum of three fiducials is required to employ $6 \mathrm{D}$ tracking. We observed early in our experience, based upon usage of the $6 \mathrm{D}$ tracking system, that fiducials must be positioned with at least $2 \mathrm{~cm}$ of separation, to accommodate the fiducial spacing threshold; have at least $1 \mathrm{~cm}$ of separation on orthogonal imaging to ensure accurate rotational corrections; and that all angles formed by the fiducial triplets have to be greater than $15^{\circ}$ for the co-linearity threshold (Figure 1B). To optimize the utility of the kilovoltage orthogonal X-ray images obtained during treatment, we use gold (density equals $19.3 \mathrm{~g} / \mathrm{ml}$ ) fiducials (10132-2, Best Medical International, Inc., Springfield, VA, USA). Each strand consists of two fiducials, each measuring $5 \mathrm{~mm}$ by $0.8 \mathrm{~mm}$, separated by a $2-\mathrm{cm}$ absorbable strand to optimize fiducial spacing.

Fiducials may be placed within the prostate via trans-rectal or trans-perineal approaches. To improve the accuracy of fiducial placement and minimize the risk of infection, we utilized the transperineal approach. Prior to fiducial placement, prostate dimensions, and volumes were determined using trans-rectal ultrasound (TRUS; Littrup et al., 1991). Next, using real time TRUS guidance, two sets of the stranded gold fiducials (a total of four fiducials), preloaded in 18 gage needles, were placed trans-perineally into the prostate with the aid of a fixed-frame guidance system (Figures 2A-C). The fiducials were placed in the peripheral zone at the left apex, right apex, left base, and right base to maximize targeting accuracy of the prostate adjacent to the prostate rectal interface. The median prostate length in this study was $4.7 \mathrm{~cm}$ (Table 1) allowing for easy placement of the $3.0-\mathrm{cm}$ strand. The left and right needles were offset by $0.5 \mathrm{~cm}$ in the $X$-axis (Figure 2A) to prevent overlap on orthogonal imaging. The procedure typically required $15 \mathrm{~min}$. Fiducial placement-related toxicities were assessed using the Common Toxicity Criteria (CTC), version 3.

\section{TREATMENT PLANNING}

MRI and CT scans for treatment planning were performed 7 days after fiducial implantation to ensure adequate tissue fixation and to allow for resolution of procedural edema/inflammation (Poggi et al., 2003; Pouliot et al., 2003; Kupelian et al., 2005). Precautions were taken to minimize prostate motion during the planning scans and treatment. Specifically, starting 5 days prior to acquisition of

\section{Table 1 | Summary of the prostate size and number of fiducials available for tracking.}

\begin{tabular}{llc}
\hline Number of patients & \multicolumn{2}{c}{88} \\
Prostate dimensions & Median & Range \\
Height $(\mathrm{mm})$ & 29 & $14-60$ \\
Width $(\mathrm{mm})$ & 46 & $29-67$ \\
Length $(\mathrm{mm})$ & 47 & $30-69$ \\
Volume $\left(\mathrm{mm}^{3}\right)$ & 32 & $11.1-143$ \\
Number of fiducials available for tracking & & \\
3: & $2(2.3 \%)$ patients \\
4: & $86(97.7 \%)$ patients
\end{tabular}




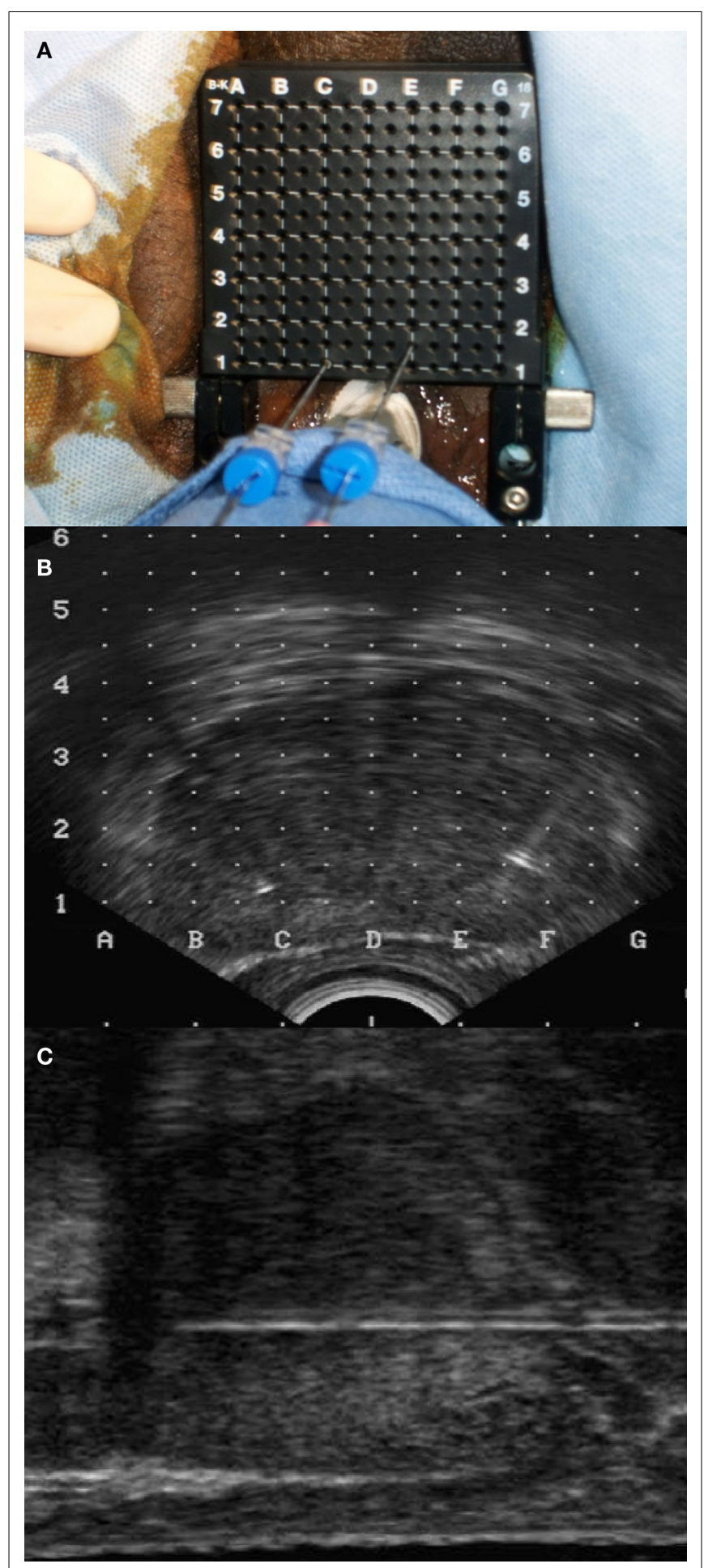

FIGURE 2 | Fiducial placement. (A) A fixed-frame system with needles is used to place fiducials under trans-rectal ultrasound guidance. (B) Axial and (C) sagittal views of ultrasound image guidance.

the planning scans until the end of treatment, patients maintained a low fiber diet to reduce intestinal gas (Smitsmans et al., 2008). Fasts were also initiated $4 \mathrm{~h}$ before both acquisition of the planning scans and each treatment session to minimize rectal movement. Enemas were performed $2 \mathrm{~h}$ prior to acquisition of the planning scans and each treatment session to minimize rectal volume. All patients were imaged and treated in the supine treatment position with a knee cushion to maximize patient comfort and limit prostate motion in response to respiration (Malone et al., 2000).

Fused thin cut CT images $(1.25 \mathrm{~mm})$ and high-resolution MR images were used for treatment planning (Figures 3A-C). MRI imaging was employed to define the target volume (Roach et al., 1996) and to aid in accurate localization of the bladder neck, membranous urethra, and penile bulb (Mclaughlin et al., 2005). Intraprostatic fiducials were employed to guide image co-registration and limit fusion errors (Parker et al., 2003). MR images were obtained on a 1.5-T scanner in a phased-array torso coil. The MRI was quickly $(<1 \mathrm{~h}$ ) followed by a CT scan to minimize anatomical changes in the rectum that may interfere with image fusion. Two MRI sequences were employed to maximize visualization of the fiducials (susceptibility-weighted gradient-echo images) and the soft tissues (axial high-resolution turbo T2-weighted spin-echo images). Treatment planning included the prostate as the gross target volume (GTV). The CTV included the prostate and the proximal seminal vesicles. The PTV equaled the CTV expanded by $3 \mathrm{~mm}$ posteriorly and $5 \mathrm{~mm}$ in all other dimensions. Treatment planning was generally completed within 1 week of imaging and subsequent treatment was initiated within a 1- to 2-week window.

\section{PRETREATMENT EVALUATION OF FIDUCIAL PLACEMENT}

A minimum of three properly placed fiducials is required to accommodate 6D tracking. Using the stranded fiducials, four fiducials are implanted which accommodates four separate fiducial triplets, although only one triplet that meets threshold criteria is required. The distances between individual fiducials (six lines) were determined for each patient to assure that the fiducial spacing threshold was met. Likewise, the angles formed by the triplets (12 angles) were determined to assure that the co-linearity threshold was met. Results from a sample patient are shown in Table 2.

\section{FIDUCIAL TRACKING}

Fiducial markers were identified in the planning CT scan and the image was converted into a digitally reconstructed radiograph (DRR; Figures 4A,B, Synthetic image). The image registration algorithm is based on alignment of the known DRR positions with the marker locations extracted from the treatment orthogonal X-ray images (Figures 4A,B, Camera image). An assessment of potential marker migration is made automatically by determining individual marker misalignment after registration (Figures 4A,B, Overlay image). Small $(<1 \mathrm{~mm})$ changes in fiducial position due to prostate deformation (Deurloo et al., 2005; Van Der Wielen et al., 2008) are allowed; however, changes in the individual fiducial marker locations that exceed a 1-mm rigid body distance threshold (Littrup et al., 1991) are assumed to have migrated from the time of the treatment planning scans and are not used for guiding treatment. The interval between successive image acquisitions and alignment corrections is adapted throughout each treatment fraction based on the intra-fraction stability of the target position (translation) and orientation (rotation). The goals of fiducial tracking are to keep the translational shifts to less than $2 \mathrm{~mm}$ and 


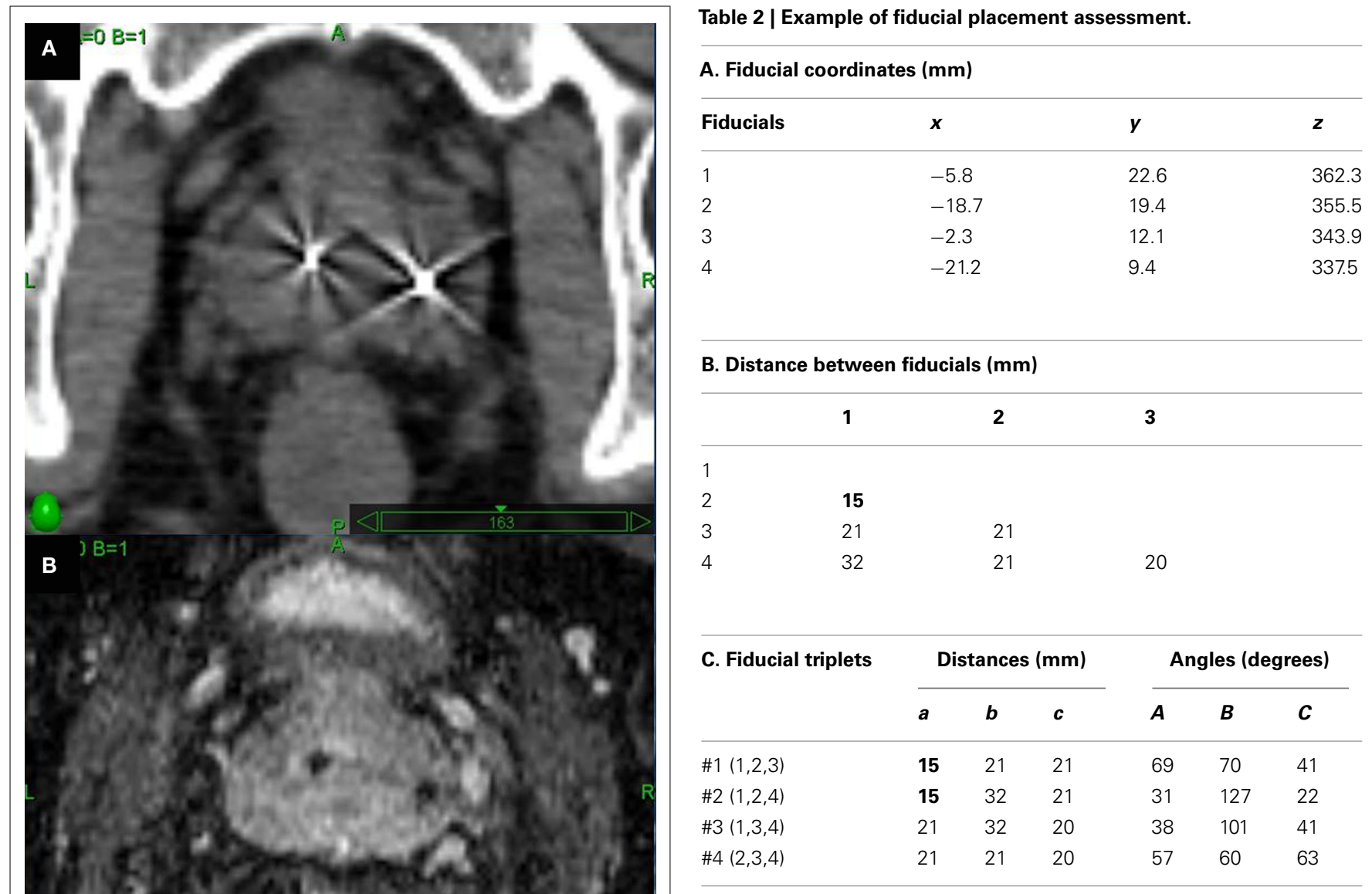

(A) The coordinates for the four implanted fiducials. (B) The calculated distances between fiducials. (C) Assessment of four possible fiducial triplets. In this case, the distance between fiducials 1 and 2 is less than the spacing threshold of $20 \mathrm{~mm}$ (bold). Thus, fiducial triplets \#1 and \#2 failed the spacing criteria. However, fiducial triplets \#3 (fiducials 1,3,4) and \#4 (fiducials 2,3,4) exceeded both the spacing and collinearity thresholds, thus allowing $6 D$ target tracking.

the rotational shifts to less than $5^{\circ}$ throughout treatment. In general, imaging is obtained every $30-60 \mathrm{~s}$ to assure sub-millimeter tracking accuracy (Xie et al., 2008). In the subset of patients with a high frequency of excursions, the treating clinician is capable of increasing the imaging frequency to maintain this accuracy.

\section{RESULTS}

From July 2008 to July 2010, 88 patients underwent trans-perineal implantation of four gold fiducials using TRUS guidance at our institution. Sixty-nine patients received CyberKnife SBRT alone (total dose 35-36.25 Gy delivered in five fractions) and 19 patients received CyberKnife SBRT (total dose 19.5 Gy delivered in three fractions) followed by IMRT (total dose of 45-50.4 Gy delivered in 25-28 fractions). Each CyberKnife SBRT treatment was typically completed in $40-60 \mathrm{~min}$. The median prostate volume was $32 \mathrm{cc}$ consistent with the patient population characteristics summarized in Table 1. There was minimal acute toxicity following the implantation procedure consisting primarily of grade 1 and 2 perineal pain lasting for $24-48 \mathrm{~h}$ which was relieved by mild analgesics. No grade 3 or higher complications per the CTC v3 criteria. 


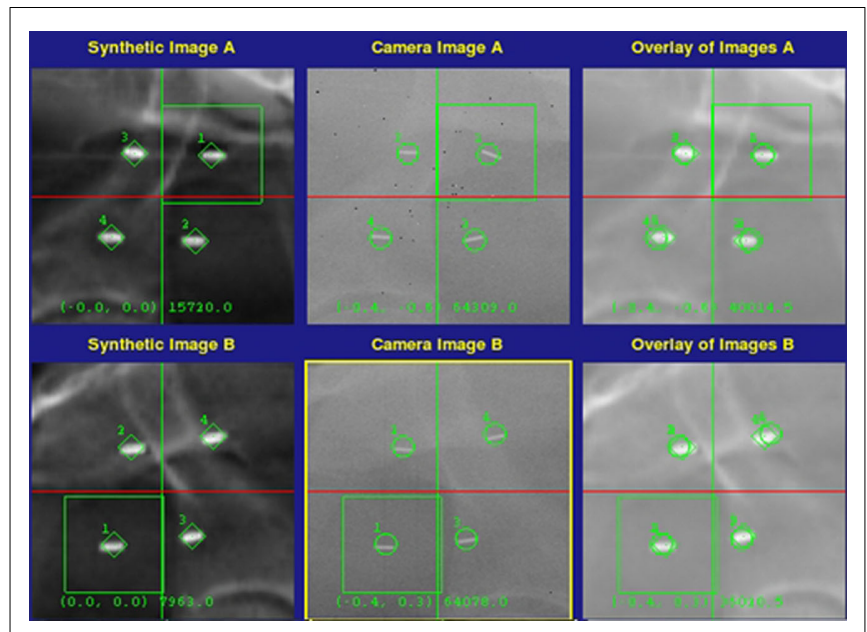

FIGURE 4 | Six-dimensional fiducial tracking for stereotactic body radiation therapy with the CyberKnife system. Shown are digitally reconstructed radiographs (left column), real time orthogonal X-ray images (middle column), and co-registered overlay images (right column).

There were no post-implant urinary tract infections or episodes of urinary retention.

Analysis of the treatment planning CT scans verified the presence of at least three fiducials in all cases. In two of the 88 patients, a single fiducial was lost between the time of fiducial placement and simulation (Table 1). Overall, $75 \%$ of the fiducial distances exceeded the $2-\mathrm{cm}$ fiducial spacing threshold and $98 \%$ of the fiducial angles exceeded the $15^{\circ}$ co-linearity threshold. There were no cases of fiducial overlap on orthogonal imaging. In $98 \%$ of cases, at least one fiducial triplet met the criteria for $6 \mathrm{D}$ correction of prostate motion. In two patients, all four possible fiducial triplets failed the spacing and/or collinearity threshold. As a result, CyberKnife treatment was limited to 3D tracking for those two patients. Fiducial tracking was also utilized for patient set-up in 19 patients treated in combination with conventional external beam radiation therapy.

\section{DISCUSSION}

The ability of dose escalation to improve disease control in clinically localized prostate cancer has been supported by several clinical studies and is accepted as a goal in prostate cancer treatment (Pollack et al., 2002; Fowler, 2005; Miles and Lee, 2008). A concern with dose escalation, however, is the possibility of increasing acute and late toxicities. For this reason, PTV margins are typically minimized thereby limiting dose to surrounding normal tissues (Dearnaley et al., 2007). Reduced PTV margins require accurate beam positioning and verification to ensure delivery of the planned dose to the target. Fiducials are accurate and reproducible surrogates for prostate location and allow for correction of both inter-fraction and intra-fraction prostate motions (Deurloo et al., 2005; Kupelian et al., 2005; Van Der Wielen et al., 2008). High-dose IMRT using fiducial-based correction of translational motion prior to treatment is well tolerated with a low incidence of acute and late gastrointestinal and genitourinary side effects (Lips et al., 2008). Such positional verification also allows the delivery of increased radiation doses without deterioration in quality of life compared with lower doses using less accurate conformal radiotherapy (Lips et al., 2007). Early results with CyberKnife SBRT suggest that the addition of 6D correction of intra-fraction prostate motion allows hypo-fractionated radiobiologic dose escalation while maintaining quality of life (Fuller et al., 2008; Friedland et al., 2009; Katz et al., 2010b; Freeman and King, 2011; Oermann et al., 2011; Jabbari et al., in press; King et al., in press).

The optimal number and location of implanted markers for image guided radiation therapy has been investigated (Kudchadker et al., 2009). In previous studies, one to six fiducials were implanted (Poggi et al., 2003; Henry et al., 2004; Moman et al., 2010). At least three properly positioned fiducials are required for translational and rotational correction with the CyberKnife system. Thus, we placed four fiducials to allow for possible loss/migration of one fiducial; an event that occurred in two of our patients. It is unclear whether the accuracy of targeted radiation therapy is improved by placing additional fiducials. However, placement of greater than four fiducials may increase the risk of fiducial mis-identification due to their close proximity (Mu et al., 2008).

Intra-fractional motion correction should accommodate further investigational dose escalation by limiting dose to normal structures. Two strategies currently exist for dealing with intrafraction motion: reducing treatment times and/or performing continuous tracking during treatment. It is well known that the extent of intra-fraction motion increases with increasing duration of treatment (Ghilezan et al., 2005; Kotte et al., 2007; Langen et al., 2008). Rotational therapy, such as volumetric modulated arc therapy (VMAC) or RapidArc (Varian Medical Systems, Palo Alto, CA, USA), can reduce treatment times to just a few minutes (Wolff et al., 2009; Aznar et al., 2010) possibly decreasing the impact of intra-fraction motion. Unfortunately, large prostate motions are not predictable and commonly occur early in the course of a treatment (Langen et al., 2008). While ignoring intra-fraction motion may be acceptable with extended fractionation regimens due to intra-fraction motion's random nature, failure to account for intra-fraction motion may lead to clinically meaningful errors with hypo-fractionated regimens (Hoogeman et al., 2008) where small prostate dose decrements could be more clinically significant (Craig et al., 2003).

The added benefit of correcting for rotational movement is the subject of investigation (Mutanga et al., 2011). Some argue that small deformations interpreted as rotations could actually increase the risk of geometric miss (Wu et al., 2007). The smaller treatment margins required for hypo-fractionation increase the importance of correcting for even small treatment errors. The appropriate PTV margins necessary to accommodate rotations are both challenging to determine and likely patient specific (Langer et al., 2005) making them difficult to apply in the clinical setting. Intra-fractional rotational correction eliminates the need for these complex and potentially large PTV expansions. When treating the prostate alone, the target volume approximates a sphere making rotational correction likely of little benefit. However, when the target volume is the prostate and the proximal seminal vesicle, rotational corrections may be important to prevent under-dosing of the seminal vesicles (Lips et al., 2009; Rijkhorst et al., 2009). 


\section{CONCLUSION}

Hypo-fractionated SBRT with 6D correction of prostate motion is a promising new treatment option for men with low- and intermediate-risk prostate cancer. Early results suggest encouraging biochemical responses with low toxicities (Fuller et al., 2008; Friedland et al., 2009; Katz et al., 2010b; Freeman and King, 2011; Oermann et al., 2011; Jabbari et al., in press; King et al., in press). $6 \mathrm{D}$ correction of intra-fraction prostate motion may be critical to obtaining such outcomes. Our experience shows

\section{REFERENCES}

Aznar, M. C., Petersen, P. M., Logadottir, A., Lindberg, H., Korreman, S. S., Kjaer-Kristoffersen, F., and Engelholm, S. A. (2010). Rotational radiotherapy for prostate cancer in clinical practice. Radiother. Oncol. 97, 480-484.

Bentel, G. C., Marks, L. B., Sherouse, G. W., Spencer, D. P., and Anscher, M. S. (1995). The effectiveness of immobilization during prostate irradiation. Int. J. Radiat. Oncol. Biol. Phys. 31, 143-148.

Chandra, A., Dong, L., Huang, E., Kuban, D. A., O'neill, L., Rosen, I., and Pollack, A. (2003). Experience of ultrasound-based daily prostate localization. Int. J. Radiat. Oncol. Biol. Phys. 56, 436-447.

Collins, S. P., Suy, S., Oermann, E., Lie, S., Yu, X., Hanscom, H., Kim, J., Sherer, B., Park, H. U., Collins, B. T., Mcgeagh, K., Dawson, N., Lynch, J. H., and Dritschilo, A. (2011). "Patient selection for robotic radiosurgery for clinically localized prostate cancer: come one, come all," in Robotic Radiosurgery: Treating Prostate Cancer and Related Genitourinary Applications, ed. L. Ponky (Heidelberg: Springer Verlag), 165-176.

Craig, T., Moiseenko, V., Battista, J., and Van Dyk, J. (2003). The impact of geometric uncertainty on hypofractionated external beam radiation therapy of prostate cancer. Int. J. Radiat. Oncol. Biol. Phys. 57, 833-842.

Crook, J. M., Raymond, Y., Salhani, D., Yang, H., and Esche, B. (1995). Prostate motion during standard radiotherapy as assessed by fiducial markers. Radiother. Oncol. 37, 35-42.

Dawson, L. A., Mah, K., Franssen, E., and Morton, G. (1998). Target position variability throughout prostate radiotherapy. Int. J. Radiat. Oncol. Biol. Phys. 42, 1155-1161.

Dearnaley, D. P., Sydes, M. R., Graham, J. D., Aird, E. G., Bottomley, D., Cowan, R. A., Huddart, R. A., Jose, C. C., Matthews, J. H., Millar, J., Moore, A. R., Morgan, R. C., Russell, J. M., Scrase, C. D., Stephens,
R. J., Syndikus, I., and Parmar, M. K. (2007). Escalated-dose versus standard-dose conformal radiotherapy in prostate cancer: first results from the MRC RT01 randomised controlled trial. Lancet Oncol. 8, 475-487.

Deurloo, K. E., Steenbakkers, R. J., Zijp, L. J., De Bois, J. A., Nowak, P. J., Rasch, C. R., and Van Herk, M. (2005). Quantification of shape variation of prostate and seminal vesicles during external beam radiotherapy. Int. J. Radiat. Oncol. Biol. Phys. 61, 228-238.

Fowler, J. F. (2005). The radiobiology of prostate cancer including new aspects of fractionated radiotherapy. Acta Oncol. 44, 265-276.

Freeman, D. E., and King, C. R. (2011). Stereotactic body radiotherapy for low-risk prostate cancer: five-year outcomes. Radiat. Oncol. 6,3 .

Friedland, J. L., Freeman, D. E., Masterson-Mcgary, M. E., and Spellberg, D. M. (2009). Stereotactic body radiotherapy: an emerging treatment approach for localized prostate cancer. Technol. Cancer Res. Treat. 8 , 387-392.

Fuller, D. B., Naitoh, J., Lee, C., Hardy, S., and Jin, H. (2008). Virtual HDR CyberKnife treatment for localized prostatic carcinoma: dosimetry comparison with HDR brachytherapy and preliminary clinical observations. Int. J. Radiat. Oncol. Biol. Phys. 70, 1588-1597.

Ghilezan, M. J., Jaffray, D. A., Siewerdsen, J. H., Van Herk, M., Shetty, A., Sharpe, M. B., Zafar Jafri, S., Vicini, F. A., Matter, R. C., Brabbins, D. S., and Martinez, A. A. (2005). Prostate gland motion assessed with cinemagnetic resonance imaging (cineMRI). Int. J. Radiat. Oncol. Biol. Phys. 62, 406-417.

Henry, A. M., Wilkinson, C., Wylie, J. P., Logue, J. P., Price, P., and Khoo, V. S. (2004). Trans-perineal implantation of radio-opaque treatment verification markers into the prostate: an assessment of procedure related morbidity, patient acceptability and accuracy. Radiother. Oncol. 73, 57-59. that with accurate fiducial placement, $6 \mathrm{D}$ correction of prostate motion with CyberKnife SBRT is feasible in the majority of patients. Investigation of additional patients with longer followup will be required to validate the clinical benefits of such an approach.

\section{ACKNOWLEDGMENTS}

We thank Etienne Lessard, Ph.D. (Accuray Incorporated) for helpful discussions.

Hoogeman, M. S., Nuyttens, J. J., Levendag, P. C., and Heijmen, B. J. (2008). Time dependence of intrafraction patient motion assessed by repeat stereoscopic imaging. Int. J. Radiat. Oncol. Biol. Phys. 70, 609-618.

Jabbari, S., Weinberg, V. K., Kaprealian, T., Hsu, I. C., Ma, L., Chuang, C., Descovich, M., Shiao, S., Shinohara, K., Roach, M. III, and Gottschalk, A. R. (in press). Stereotactic body radiotherapy as monotherapy or post-external beam radiotherapy boost for prostate cancer: technique, early toxicity, and PSA response. Int. J. Radiat. Oncol. Biol. Phys.

Kaiser, A., Schultheiss, T. E., Wong, J. Y., Smith, D. D., Han, C., Vora, N. L., Pezner, R. D., Chen, Y. J., and Radany, E. H. (2006). Pitch, roll, and yaw variations in patient positioning. Int. J. Radiat. Oncol. Biol. Phys. 66, 949-955.

Katz, A., Santoro, M., Ashley, R., Diblasio, F., and Witten, M. (2010a). Stereotactic body radiotherapy as boost for organ-confined prostate cancer. Technol. Cancer Res. Treat. 9, 575-582.

Katz, A. J., Santoro, M., Ashley, R., Diblasio, F., and Witten, M. (2010b). Stereotactic body radiotherapy for organ-confined prostate cancer. BMC Urol. 10, 1. doi:10.1186/1471-2490-10-1

Kilby, W., Dooley, J., Kuduvalli, G., Sayeh, S., and Maurer, C. R. J. (2010). The CyberKnife robotic radiosurgery system in 2010. Technol. Cancer Res. Treat. 9, 433-452.

King, C. R., Brooks, J. D., Gill, H., and Presti, J. C. Jr. (in press). Longterm outcomes from a prospective trial of stereotactic body radiotherapy for low-risk prostate cancer. Int. J. Radiat. Oncol. Biol. Phys.

Kotte, A. N., Hofman, P., Lagendijk, J. J., Van Vulpen, M., and Van Der Heide, U. A. (2007). Intrafraction motion of the prostate during external-beam radiation therapy: analysis of 427 patients with implanted fiducial markers. Int. J. Radiat. Oncol. Biol. Phys. 69, 419-425.
Kudchadker, R. J., Lee, A. K., Yu, Z. H., Johnson, J. L., Zhang, L., Zhang, Y., Amos, R. A., Nakanishi, H., Ochiai, A., and Dong, L. (2009). Effectiveness of using fewer implanted fiducial markers for prostate target alignment. Int. J. Radiat. Oncol. Biol. Phys. 74, 1283-1289.

Kupelian, P. A., Langen, K. M., Willoughby, T. R., Zeidan, O. A., and Meeks, S. L. (2008). Imageguided radiotherapy for localized prostate cancer: treating a moving target. Semin. Radiat. Oncol. 18, 58-66.

Kupelian, P. A., Willoughby, T. R., Meeks, S. L., Forbes, A., Wagner, T., Maach, M., and Langen, K. M. (2005). Intraprostatic fiducials for localization of the prostate gland: monitoring intermarker distances during radiation therapy to test for marker stability. Int. J. Radiat. Oncol. Biol. Phys. 62, 1291-1296.

Langen, K. M., Willoughby, T. R., Meeks, S. L., Santhanam, A., Cunningham, A., Levine, L., and Kupelian, P. A. (2008). Observations on real-time prostate gland motion using electromagnetic tracking. Int. J. Radiat. Oncol. Biol. Phys. 71, 1084-1090.

Langer, M. P., Papiez, L., Spirydovich, S., and Thai, V. (2005). The need for rotational margins in intensitymodulated radiotherapy and a new method for planning target volume design. Int. J. Radiat. Oncol. Biol. Phys. 63, 1592-1603.

Li, J. S., Jin, L., Pollack, A., Horwitz, E. M., Buyyounouski, M. K., Price, R. A. Jr., and Ma, C. M. (2009). Gains from real-time tracking of prostate motion during external beam radiation therapy. Int. J. Radiat. Oncol. Biol. Phys. 75, 1613-1620.

Lips, I., Dehnad, H., Kruger, A. B., Van Moorselaar, J., Van Der Heide, U., Battermann, J., and Van Vulpen, M. (2007). Health-related quality of life in patients with locally advanced prostate cancer after 76 Gy intensity-modulated radiotherapy vs. 70 Gy conformal radiotherapy in a prospective and longitudinal study. Int. J. Radiat. Oncol. Biol. Phys. 69, 656-661. 
Lips, I. M., Dehnad, H., Van Gils, C. H., Boeken Kruger, A. E., Van Der Heide, U. A., and Van Vulpen, M. (2008). High-dose intensitymodulated radiotherapy for prostate cancer using daily fiducial markerbased position verification: acute and late toxicity in 331 patients. Radiat. Oncol. 3, 15.

Lips, I. M., Van Der Heide, U. A., Kotte, A. N., Van Vulpen, M., and Bel, A. (2009). Effect of translational and rotational errors on complex dose distributions with off-line and on-line position verification. Int. J. Radiat. Oncol. Biol. Phys. 74, 1600-1608.

Littrup, P. J., Williams, C. R., Egglin, T. K., and Kane, R. A. (1991). Determination of prostate volume with transrectal US for cancer screening. Part II. Accuracy of in vitro and in vivo techniques. Radiology 179, 49-53.

Litzenberg, D. W., Balter, J. M., Hadley, S. W., Sandler, H. M., Willoughby, T. R., Kupelian, P. A., and Levine, L. (2006). Influence of intrafraction motion on margins for prostate radiotherapy. Int. J. Radiat. Oncol. Biol. Phys. 65, 548-553.

Malone, S., Crook, J. M., Kendal, W. S., and Szanto, J. (2000). Respiratoryinduced prostate motion: quantification and characterization. Int. J. Radiat. Oncol. Biol. Phys. 48, 105-109.

Mclaughlin, P. W., Troyer, S., Berri, S., Narayana, V., Meirowitz, A., Roberson, P. L., and Montie, J. (2005). Functional anatomy of the prostate: implications for treatment planning. Int. J. Radiat. Oncol. Biol. Phys. 63, 479-491.

Miles, E. F., and Lee, W. R. (2008). Hypofractionation for prostate cancer: a critical review. Semin. Radiat. Oncol. 18, 41-47.

Moman, M. R., Van Der Heide, U. A., Kotte, A. N., Van Moorselaar, R. J., Bol, G. H., Franken, S. P., and Van Vulpen, M. (2010). Long-term experience with transrectal and transperineal implantations of fiducial gold markers in the prostate for position verification in external beam radiotherapy; feasibility, toxicity and quality of life. Radiother. Oncol. 96, 38-42.

Moseley, D. J., White, E. A., Wiltshire, K. L., Rosewall, T., Sharpe, M. B., Siewerdsen, J. H., Bissonnette, J. P., Gospodarowicz, M., Warde, P., Catton, C. N., and Jaffray, D. A. (2007). Comparison of localization performance with implanted fiducial markers and cone-beam computed tomography for on-line image-guided radiotherapy of the prostate. Int. J. Radiat. Oncol. Biol. Phys. 67, 942-953.

Mu, Z., Fu, D., and Kuduvalli, G. (2008). A probabilistic framework based on hidden Markov model for fiducial identification in image-guided radiation treatments. IEEE Trans. Med. Imaging 27, 1288-1300.

Mutanga, T. F., De Boer, H. C., Van Der Wielen, G. J., Hoogeman, M. S., Incrocci, L., and Heijmen, B. J. (2011). Margin evaluation in the presence of deformation, rotation, and translation in prostate and entire seminal vesicle irradiation with daily marker-based setup corrections. Int. J. Radiat. Oncol. Biol. Phys. 81, 1160-1167.

Oermann, E., Hanscom, H., Lei, S., Suy, S., Collins, B., Batipps, G., Mcgeagh, K., Jha, R., Dawson, N., Dritschilo, A., Lynch, J., and Collins, S. P. (2010). A pilot study of intensity modulated radiation therapy plus a hypofractionated stereotactic body radiation therapy boost for the treatment of intermediate to high risk prostate cancer. Technol. Cancer Res. Treat. 9, 453-462.

Oermann, E. K., Suy, S., Hanscom, H. N., Kim, J. S., Lei, S., Yu, X., Zhang, G., Ennis, B., Rohan, J. P., Piel, N., Sherer, B. A., Borum, D., Chen, V. J., Batipps, G. P., Constantinople, N. L., Dejter, S. W., Bandi, G., Pahira, J., Mcgeagh, K. G., Adams-Campbell, L., Jha, R., Dawson, N. A., Collins, B. T., Dritschilo, A., Lynch, J. H., and Collins, S. P. (2011). Low incidence of new biochemical and clinical hypogonadism following hypofractionated stereotactic body radiation therapy (SBRT) monotherapy for low- to intermediate-risk prostate cancer. J. Hematol. Oncol. 4, 12.

Parker, C. C., Damyanovich, A., Haycocks, T., Haider, M., Bayley, A., and Catton, C. N. (2003). Magnetic resonance imaging in the radiation treatment planning of localized prostate cancer using intra-prostatic fiducial markers for computed tomography co-registration. Radiother. Oncol. 66, 217-224.

Poggi, M. M., Gant, D. A., Sewchand, W., and Warlick, W. B. (2003). Marker seed migration in prostate localization. Int. J. Radiat. Oncol. Biol. Phys. 56, 1248-1251.

Pollack, A., Zagars, G. K., Starkschall, G., Antolak, J. A., Lee, J. J., Huang, E.,
Von Eschenbach, A. C., Kuban, D. A., and Rosen, I. (2002). Prostate cancer radiation dose response: results of the M. D. Anderson phase III randomized trial. Int. J. Radiat. Oncol. Biol. Phys. 53, 1097-1105.

Pouliot, J., Aubin, M., Langen, K. M. Liu, Y. M., Pickett, B., Shinohara, K. and Roach, M. III. (2003). (Non)migration of radiopaque markers used for on-line localization of the prostate with an electronic portal imaging device. Int. J. Radiat. Oncol. Biol. Phys. 56, 862-866.

Rijkhorst, E. J., Lakeman, A., Nijkamp, J., De Bois, J., Van Herk, M., Lebesque, J. V., and Sonke, J. J. (2009). Strategies for online organ motion correction for intensity-modulated radiotherapy of prostate cancer: prostate, rectum, and bladder dose effects. Int. J. Radiat. Oncol. Biol. Phys. 75, 1254-1260.

Roach, M. III, Faillace-Akazawa, P., Malfatti, C., Holland, J., and Hricak, H. (1996). Prostate volumes defined by magnetic resonance imaging and computerized tomographic scans for three-dimensional conformal radiotherapy. Int. J. Radiat. Oncol. Biol. Phys. 35, 1011-1018.

Smitsmans, M. H., Pos, F. J., De Bois, J., Heemsbergen, W. D., Sonke, J. J., Lebesque, J. V., and Van Herk, M. (2008). The influence of a dietary protocol on cone beam CT-guided radiotherapy for prostate cancer patients. Int. J. Radiat. Oncol. Biol. Phys. 71, 1279-1286.

Ullman, K. L., Ning, H., Susil, R. C., Ayele, A., Jocelyn, L., Havelos, J., Guion, P., Xie, H., Li, G., Arora, B. C., Cannon, A., Miller, R. W., Coleman, C. N., Camphausen, K., and Menard, C. (2006). Intra- and interradiation therapist reproducibility of daily isocenter verification using prostatic fiducial markers. Radiat. Oncol. 1, 2.

Van Der Wielen, G. J., Mutanga, T. F., Incrocci, L., Kirkels, W. J., Vasquez Osorio, E. M., Hoogeman, M. S., Heijmen, B. J., and De Boer, H. C. (2008). Deformation of prostate and seminal vesicles relative to intraprostatic fiducial markers. Int. J. Radiat. Oncol. Biol. Phys. 72, 1604-1611.

Wolff, D., Stieler, F., Welzel, G., Lorenz, F., Abo-Madyan, Y., Mai, S., Herskind, C., Polednik, M., Steil, V., Wenz, F., and Lohr, F. (2009). Volumetric modulated arc therapy (VMAT) vs. serial tomotherapy, step-and-shoot IMRT and 3D-conformal RT for treatment of prostate cancer. Radiother. Oncol.93, 226-233.

Wu, X., Fu, D., De La Zerda, A., Bossart, E., Shao, H., Both, J., Nikesch, W. Huang, Z., Markoe, A., and Schwade, J. (2007). "Patient alignment and target tracking in radiosurgery of softtissue tumors using combined fiducial and skeletal structures tracking techniques," in Robotic Radiosurgery: Treating Tumors that Move with Respiration, ed. H. Urschel (Berlin, Heidelberg: Springer), 105-110.

Xie, Y., Djajaputra, D., King, C. R., Hossain, S., Ma, L., and Xing, L. (2008). Intrafractional motion of the prostate during hypofractionated radiotherapy. Int. J. Radiat. Oncol. Biol. Phys. 72, 236-246.

Zelefsky, M. J., Fuks, Z., Hunt, M., Yamada, Y., Marion, C., Ling, C. C., Amols, H., Venkatraman, E. S., and Leibel, S. A. (2002). Highdose intensity modulated radiation therapy for prostate cancer: early toxicity and biochemical outcome in 772 patients. Int. J. Radiat. Oncol. Biol. Phys. 53, 1111-1116.

Conflict of Interest Statement: Brian T. Collins and S. P. Collins serve as a clinical consultants to Accuray Inc. The other authors declare that they have no competing interests.

Received: 15 September 2011; paper pending published: 14 October 2011; accepted: 14 November 2011; published online: 08 December 2011.

Citation: Lei S, Piel N, Oermann EK, Chen V, Ju AW, Dahal KN, Hanscom HN, Kim JS, Yu X, Zhang G, Collins BT, Jha $R$, Dritschilo A, Suy $S$ and Collins SP (2011) Six-dimensional correction of intra-fractional prostate motion with CyberKnife stereotactic body radiation therapy. Front. Oncol. 1:48. doi: 10.3389/fonc. 2011.00048

This article was submitted to Frontiers in Radiation Oncology, a specialty of Frontiers in Oncology.

Copyright (c) 2011 Lei, Piel, Oermann, Chen, Ju, Dahal, Hanscom, Kim, Yu, Zhang, Collins, Jha, Dritschilo, Suy and Collins. This is an open-access article distributed under the terms of the Creative Commons Attribution Non Commercial License, which permits noncommercial use, distribution, and reproduction in other forums, provided the original authors and source are credited. 